\title{
Complications and mortality in patients with schizophrenia and diabetes: population-based cohort study
}

\author{
Chi-Shin Wu, Mei-Shu Lai and Susan Shur-Fen Gau
}

\section{Background}

The long-term outcome of patients with both diabetes and schizophrenia remains unclear.

\section{Aims \\ To explore whether having schizophrenia increases the risk of advanced complications and mortality in people with diabetes.}

\section{Method}

This is a population-based matched cohort study using Taiwan's National Health Insurance Research Database. A total of 11247 participants with diabetes and schizophrenia and 11247 participants with diabetes but not schizophrenia were enrolled. We used Cox proportional hazard models to determine the effect of schizophrenia on macrovascular and microvascular complications, and all-cause mortality.

\section{Results}

The adjusted hazard ratios were 1.49 (95\% Cl 1.32-1.68) for macrovascular complications, $1.05(95 \% \mathrm{Cl} 0.91-1.21)$ for microvascular complications and 3.68 (95\% Cl 3.21-4.22) for all-cause mortality in patients with diabetes and schizophrenia compared with those patients with diabetes but not schizophrenia.

\section{Conclusions}

Patients with both diabetes and schizophrenia had an increased risk of macrovascular complications and all-cause mortality but did not have statistically significant elevated risk of microvascular complications.

\section{Declaration of interest}

None.

\section{Copyright and usage}

(c) The Royal college of Psychiatrists 2015.
Diabetes mellitus is a common chronic metabolic disorder characterised by elevated blood glucose. Prolonged hyperglycaemia may induce the macrovascular and microvascular complications of diabetes. ${ }^{1}$ Intensive glycaemic control, as well as lifestyle intervention, is recommended for diabetes management. ${ }^{2}$ Patients with schizophrenia have a significantly higher prevalence of diabetes mellitus than the general population. ${ }^{3-5}$ Factors associated with developing diabetes mellitus, such as heritability, an unhealthy lifestyle and antipsychotic treatment, might further influence disease progression and the long-term adverse outcomes of diabetes. ${ }^{6-8}$ However, whether schizophrenia is associated with poor glycaemic control remains inconclusive. Three crosssectional studies showed that the haemoglobin $\mathrm{A}_{1 \mathrm{c}}\left(\mathrm{Hb}_{1 \mathrm{c}}\right)$ level in patients with schizophrenia was similar to or lower than that in people without severe mental illnesses. ${ }^{9-11}$ In contrast, a retrospective cohort study showed that patients with schizophrenia were more likely to be admitted to hospital or visit emergency departments for hyperglycaemia or hypoglycaemia. ${ }^{12}$ Studies exploring long-term diabetes complications in patients with schizophrenia have revealed mixed results. ${ }^{13,14}$ The discrepant findings might be partially explained by enrolling patients with prevalent diabetes without controlling for duration of diabetes, ${ }^{13,14}$ small sample size ${ }^{13}$ and short length of follow-up. ${ }^{14}$ Therefore, we conducted a matched cohort study using a nationwide claims database to explore the association between schizophrenia and advanced diabetes complications and mortalities in patients with newly diagnosed diabetes.

\section{Method}

\section{Data source}

This matched cohort study from 1998 to 2011 was carried out based on the Taiwan's National Health Insurance Research
Database (NHIRD). ${ }^{15}$ Taiwan launched a single-payer National Health Insurance (NHI) programme, covering up to $98 \%$ of the Taiwanese population on 1 March 1995. The NHIRD was derived from the original reimbursement claims. The database includes patients' demographic characteristics, diagnoses, procedures and prescription claims data. The validation of diagnoses of major diseases in the NHIRD, such as diabetes, stroke and acute coronary syndrome, has been well-established. ${ }^{16-18}$

Given we could not assess the whole database of the NHIRD, we used the Psychiatric Inpatient Medical Claims (PIMC) and the Longitudinal Health Insurance Database 2005 (LHID2005), which are subsets of the NHIRD, to identify patients with diabetes with and without schizophrenia respectively. The PIMC contains all beneficiaries with at least one psychiatric hospital admission record and one discharge diagnosis of psychiatric disorder between 1996 and 2007, which is derived from the whole population in Taiwan $(n=187117)$. The claims data were extended to 2011 for this study. The LHID2005 contains all the original claims data of 1000000 beneficiaries, randomly sampled from the year 2005 Registry for Beneficiaries of the NHIRD. The distribution of gender and age of the sampled participants in the LHID2005 did not differ significantly from that of the general population. This study was approved by the Research Ethics Review Committee of Far Eastern Memorial Hospital before implementation.

\section{Study population}

We assembled a cohort of patients with newly diagnosed diabetes. The index date, the date of cohort entry, was defined as the date of the first diagnosis of diabetes. Patients with diabetes included in this study must have had at least three ambulatory claims or one in-patient discharge diagnosis of diabetes (ICD-9-CM code: 
250.x $)^{19}$ between 1998 and 2011. The validation of this definition of diabetes showed a $96.9 \%$ sensitivity and $93.9 \%$ positive predictive value in a study using a questionnaire assessment of patients with diabetes from the NHIRD. ${ }^{17,20}$

Prevalent cases of individuals with a diagnosis of diabetes before 1998 or those with less than a 1-year observational period before diabetes was diagnosed were excluded from the study. Patients with newly diagnosed diabetes aged $<18$ years on the index date were excluded. We also excluded patients who had any ambulatory or in-patient record of the study end-point (microvascular and macrovascular complications) before the index date. The details of the data refinement procedure to identify the cohorts of patients with both diabetes and schizophrenia (diabetes+schizophrenia group) and those with diabetes but without schizophrenia (comparison group) are shown in Fig. 1 and described below.

\section{Cohort of patients with diabetes and schizophrenia}

A total of 14740 individuals newly diagnosed with diabetes and who had schizophrenia were identified from PIMC between the years 1998 and 2011. The included patients must have had a diagnosis of schizophrenia (ICD9-CM code: 295.x) before the first date of diabetes diagnosis. To ensure the validity of the schizophrenia diagnosis, patients with schizophrenia should have been confirmed by an in-patient diagnosis of schizophrenia. After excluding those aged $<18$ years and those with pre-existing complications, a total of 12128 patients with diabetes and with schizophrenia were identified.

\section{Comparison group of patients with diabetes but without schizophrenia}

We identified 62183 eligible comparison individuals, who had no pre-existing complications, were aged $\geqslant 18$ years and no diagnosis
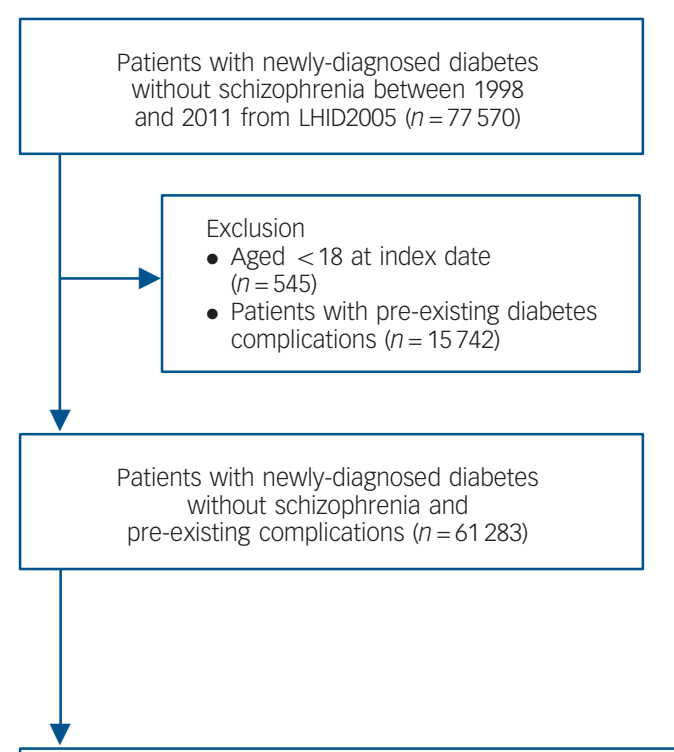

Matched by age (the year of birth), gender and the year of index date

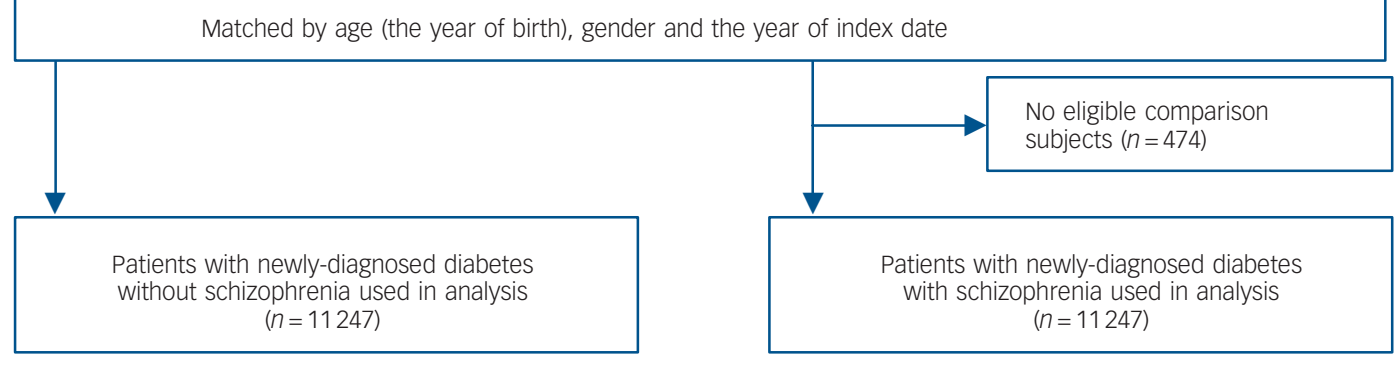

Fig. 1 Flow chart of selection of study population. of schizophrenia before the first date of diabetes diagnosis. For each patient in the diabetes+schizophrenia group, we randomly selected one comparison individual (patient with diabetes but without schizophrenia) matched by age (the year of birth), gender and year of diabetes diagnosis. The matched comparison patients were not selected again once they had been matched. Since all comparison individuals participated in the NHI programme in 2005, patients with diabetes and with schizophrenia who were not NHI beneficiaries in 2005 were excluded. When there were no eligible comparison individuals, an equal number of patients with both diabetes and with schizophrenia were excluded $(n=474)$. We finally identified 11274 patients with both diabetes and schizophrenia (diabetes+schizophrenia group) and 11274 patients with diabetes but without schizophrenia (comparison group).

\section{Main outcome measures}

Advanced complications of diabetes

We defined the advanced macrovascular and microvascular complications of diabetes based on the ICD-9-CM diagnostic and procedure code and NHI procedure code from the ambulatory and in-patient claims records. Macrovascular complications included admission to hospital for ischemic heart disease (ICD-9-CM: 410.x-414.x), stroke (ICD-9-CM: 430 -438.x) and peripheral vascular disease (ICD-9-CM: 443.9 or 440.2) with stent insertion, vascular shunt or bypass or vessel repair procedure (ICD-9-CM: 00.55, 39.23, 39.24, 39.25, 39.26 39.29, 39.50, 39.51, $39.52,39.54,39.56,39.57,39.58,39.59$ or 39.71$)$.

Microvascular complication was defined as the composite of diabetic retinopathy (ICD-9-CM: 250.5x, 362.01, or 362.02) with

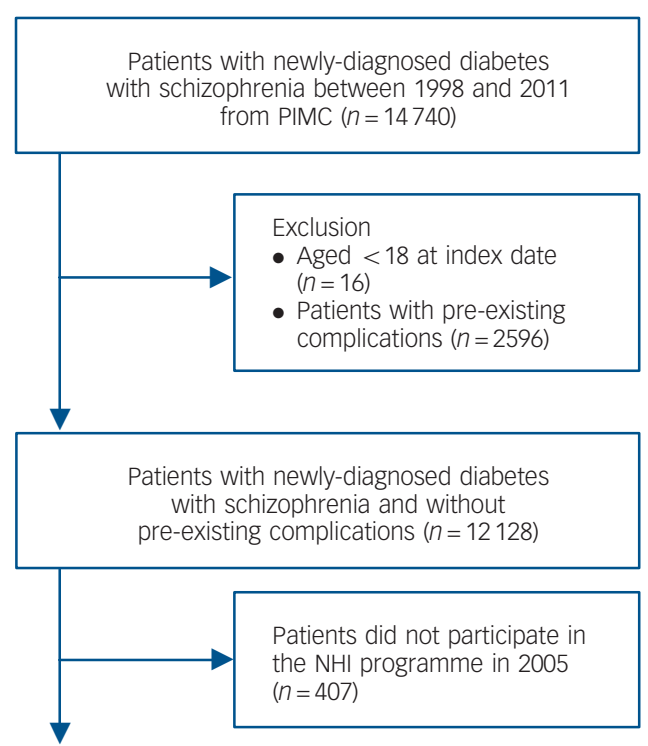

Patients did not participate in the $\mathrm{NHI}$ programme in 2005 
laser photocoagulation (NHI procedure code: $86206 \mathrm{~B}$ or $86207 \mathrm{~B}$ ) or vitrectomy (NHI procedure code: $60003 \mathrm{C}$ or $60004 \mathrm{C}$ ), blindness (ICD-9-CM: 369.x), end-stage renal disease (ICD-9-CM: 585.5585.6 or 586) with dialysis, vessel operations for haemodialysis or kidney transplantation (ICD-9-CM: 39.27, 39.42, 39.43, $39.49,39.50,39.53,39.93,39.94,39.95,54.98$ or $55.6 x$ ), admission to hospital for diabetic foot infection (ICD-9-CM: 681.1x, 682.6 or 682.7) or lower extremity amputations (ICD-9-CM procedure code: $84.1 \mathrm{x}$ ). Study cohorts were followed up until the date of the occurrence of a study outcome, death or the end of 2011.

\section{All-cause mortality}

We conducted a separate analysis for the hazard ratio (HR) of all-cause mortality. The date of death was identified from the discharge date of in-patient death, the date of withdrawal from the NHI programme or the death record in the Registry for Catastrophic Illness Patients, which is a subset of the NHIRD and shared a common identifier with all data-sets in the NHIRD. The death was further confirmed by the follow-up claims. If patients had further out-patient or in-patient visits after the ascertained death date, the death was excluded. Both the diabetes+ schizophrenia and the comparison groups participated in the NHI programme in 2005; therefore, mortality would not have occurred before 2005. If the index date of the study individual was prior to 2005, the date of cohort entry was defined as 1 January 2005. Study groups were followed up until death or the end of 2011.

\section{Covariates assessment}

In addition to matching patients by age (the year of birth), gender and the year of diabetes diagnosis, several potential confounders that might be associated with both diabetes complications and schizophrenia were examined. These factors, which were assessed in the year before the index date, included comorbid conditions of hypertension, dyslipidaemia, chronic pulmonary disease, chronic liver disease, malignancy, major depressive disorder, anxiety disorder, alcohol- or substance-related disorder and exposure to medication, including angiotensin converting-enzyme inhibitor/angiotensin II receptor blockers (ACEI/ARBs), beta blockers, calcium channel blockers, diuretics, lipid-lowering agents, non-steroidal anti-inflammatory drugs (NSAIDs) and antidepressants. To control for medical accessibility, we assessed health system utilisation, including the number of psychiatric and non-psychiatric out-patient visits, and hospital admissions to psychiatric and non-psychiatric wards in the year prior to the index date.

\section{Statistical analysis}

The baseline characteristics of patients with diabetes with and without schizophrenia are described by number and percentage. Differences in the characteristics in these two patient groups were compared using $\chi^{2}$-test. We used Kaplan-Meier event-free survival curves to compare the three outcomes: macrovascular diabetes complications, microvascular diabetes complications and all-cause mortality, among the diabetes+schizophrenia group and the comparison group and tested the difference using the log-rank test. With adjustment for all above-mentioned potential confounding factors, we used separate multivariate Cox proportional hazards models to estimate the hazard ratios of schizophrenia for the three outcomes. The proportional hazards assumption for schizophrenia, which was tested using the Kolmogorov-type supremum test, was not violated in analyses for macrovascular complications $(P=0.07)$, microvascular complications $(P=0.08)$ and all-cause mortality $(P=0.45) .^{21}$
To assess whether the risk of diabetes complications and mortality varied across patient characteristics, we conducted further analyses stratifying various characteristics of the patients, including age, gender and presence of cardiometabolic risk factors (hypertension or dyslipidaemia). Furthermore, we included the interaction terms in the multivariate model to determine the modifying effects of the patients' characteristics on the risks of diabetes complications and mortality.

We conducted post hoc analyses to assess the quality of diabetes care, which is a potential mediator contributing to the link between schizophrenia and the outcome of diabetes. The quality indicators of diabetes care were anti-diabetic medication adherence and the rates of receipt for the following tests: fasting blood glucose, $\mathrm{HbA}_{1 \mathrm{C}}$, lipid profiles, serum creatinine and urine protein, and retina examinations during the follow-up period. The 1-year weighted rate of receipt for each test was calculated using the total number of tests that patients received divided by the years of follow-up period. We assumed a rate of equal to or more than once per year for each test as an indicator of good quality of diabetes care. In addition, we assessed anti-diabetic medication adherence by using the medication process ratio (MPR), ${ }^{22}$ which was defined as the sum of the days' supply of anti-diabetic medications divided by follow-up periods. Good anti-diabetic adherence was defined as a MPR $\geqslant 0.8$. Multivariate logistic regression models were used to examine the difference in quality of care between the two groups, with adjustment for the above-mentioned covariates. All statistical analyses were conducted with SAS version 9.2. The statistical significance of relationships was assessed using $95 \%$ confidence intervals or $P<0.05$.

\section{Results}

The baseline characteristics of the diabetes+schizophrenia and the comparison groups are detailed in Table 1. The mean age at diabetes diagnosis was 45.7 years $($ s.d. $=11.7)$. Mean duration of follow-up for diabetes complications was 5.4 years (s.d.=3.7). Overall, the diabetes+schizophrenia group had a higher prevalence of dyslipidaemia, chronic pulmonary disease, chronic liver disease, major depressive disorder, anxiety disorders and alcohol- or substance-related disorders than the comparison group. In addition, the diabetes+schizophrenia group were more likely to use beta blockers and antidepressants, but were less likely to use ACEI/ARBs, calcium channel blockers, diuretics and NSAIDs. Regarding health system utilisation, the diabetes+schizophrenia group had more overall admissions to hospital $\left(\chi^{2}=2014.0\right.$, d.f. $=1, P<0.001)$ and out-patient visits $\left(\chi^{2}=1437.9\right.$, d.f. $=2$, $P<0.001)$ but fewer non-psychiatric out-patient visits $\left(\chi^{2}=296.2\right.$, d.f. $\left.=1, P<0.001\right)$ than the comparison group.

The incidence rate per 1000 person-years was 18.2 for macrovascular complications, 13.0 for microvascular complications and 26.6 for all-cause mortality in the diabetes+schizophrenia group (Table 2). In the comparison group, the incidence rates were 14.2, 11.2 and 7.5 per 1000 person-years for macrovascular complications, microvascular complications and all-cause mortality respectively. The Kaplan-Meier event-free survival curves showed the elevated risk for macrovascular complications (the log-rank $P<0.001)$, microvascular complications $(P=0.005)$ and all-cause mortality $(P<0.001)$ among the diabetes+schizophrenia group (Fig. 2). The adjusted hazard ratios of schizophrenia were 1.49 (95\% CI 1.32-1.68) for macrovascular complications and 3.68 (95\% CI 3.21-4.22) for all-cause mortality. However, the risk of schizophrenia for microvascular complications was not statistically significant after adjusting for potential confounding factors (adjusted HR $=1.05,95 \%$ CI 0.91-1.21). The hazard ratios with 


\begin{tabular}{|c|c|c|c|}
\hline & \multicolumn{2}{|l|}{$n(\%)$} & \multirow[b]{2}{*}{$P$} \\
\hline & Diabetes+schizophrenia group $(n=11247)$ & Comparison group $(n=11247)$ & \\
\hline \multicolumn{4}{|l|}{ Age, years } \\
\hline $18-44$ & $5493(48.8)$ & $5493(48.8)$ & \\
\hline $45-64$ & $5026(44.7)$ & $5026(44.7)$ & \\
\hline$\geqslant 65$ & $728(6.5)$ & $728(6.5)$ & \\
\hline Male gender & $5720(50.9)$ & $5720(50.9)$ & \\
\hline \multicolumn{4}{|l|}{ Comorbidity } \\
\hline Hypertension & 3269 (29.1) & 3325 (29.6) & 0.41 \\
\hline Dyslipidaemia & 2737 (24.3) & 2575 (22.9) & 0.01 \\
\hline Chronic liver diseases & 1635 (14.5) & $1266(11.3)$ & $<0.001$ \\
\hline Chronic pulmonary diseases & $2434(21.6)$ & $1715(15.2)$ & $<0.001$ \\
\hline Malignancy & $335(3.0)$ & $334(3.0)$ & 0.97 \\
\hline Major depressive disorders & 3299 (29.3) & $247(2.2)$ & $<0.001$ \\
\hline Anxiety disorders & 3511 (31.2) & $1310(11.6)$ & $<0.001$ \\
\hline Alcohol or substance use & 1505 (13.4) & $341(3.0)$ & $<0.001$ \\
\hline \multicolumn{4}{|l|}{ Medication use } \\
\hline ACEI/ARB & $1002(8.9)$ & $1472(13.1)$ & $<0.001$ \\
\hline Beta blocker & 4194 (37.3) & $2173(19.3)$ & $<0.0001$ \\
\hline Calcium channel blocker & $1640(14.6)$ & $2071(18.4)$ & $<0.001$ \\
\hline Diuretics & $1005(8.9)$ & $1174(10.4)$ & $<0.001$ \\
\hline Lipid-lowering agent & $1011(9.0)$ & $976(8.7)$ & 0.41 \\
\hline NSAID & $6475(57.6)$ & 8195 (72.9) & $<0.001$ \\
\hline Antidepressants & $3053(27.1)$ & $590(5.2)$ & $<0.001$ \\
\hline \multicolumn{4}{|l|}{ Healthy system utilisation } \\
\hline Number of non-psychiatric out-patient visits & & & $<0.001$ \\
\hline$<10$ & $5187(46.1)$ & 3975 (35.3) & \\
\hline $10-19$ & $2566(22.8)$ & $3399(30.2)$ & \\
\hline$\geqslant 20$ & $3494(31.1)$ & 3873 (34.4) & \\
\hline Number of psychiatric out-patient visits & & & $<0.001$ \\
\hline$<10$ & 4872 (43.3) & $11147(99.1)$ & \\
\hline $10-19$ & $4504(40.0)$ & $84(0.7)$ & \\
\hline$\geqslant 20$ & $1871(16.6)$ & $16(0.1)$ & \\
\hline \multicolumn{4}{|l|}{ Admission to hospital } \\
\hline Non-psychiatric & $1492(13.3)$ & $1151(10.2)$ & $<0.001$ \\
\hline Psychiatric & $3053(27.1)$ & $19(0.2)$ & $<0.001$ \\
\hline
\end{tabular}

\begin{tabular}{|c|c|c|c|}
\hline & \multicolumn{2}{|c|}{ Complications } & \multirow[b]{2}{*}{ All-cause mortality } \\
\hline & Macrovascular & Microvascular & \\
\hline \multicolumn{4}{|l|}{ Diabetes+schizophrenia group } \\
\hline Event/person-years & 1083/59637 & $791 / 60880$ & 1330/50053 \\
\hline Incidence per 1000 person-year $(95 \% \mathrm{Cl})$ & $18.2(17.1-19.2)$ & $13.0(12.1-13.9)$ & $26.6(25.1-28.0)$ \\
\hline \multicolumn{4}{|l|}{ Comparison group } \\
\hline Event/person-years & $888 / 62386$ & 709/63100 & 392/52089 \\
\hline Incidence per 1000 person-year $(95 \% \mathrm{Cl})$ & $14.2(13.3-15.2)$ & $11.2(10.4-12.1)$ & $7.5(6.8-8.3)$ \\
\hline \multicolumn{4}{|l|}{ Adjusted hazard ratio of schizophrenia $(95 \%$ Cl) } \\
\hline All patients & $1.49(1.32-1.68)$ & $1.05(0.91-1.21)$ & $3.68(3.21-4.22)$ \\
\hline \multicolumn{4}{|l|}{ Subgroup analysis } \\
\hline \multicolumn{4}{|l|}{ Age group, years } \\
\hline 18-44 & $1.47(1.18-1.83)$ & $0.90(0.72-1.12)$ & $3.04(2.39-3.85)$ \\
\hline $45-64$ & $1.31(1.11-1.55)$ & $1.07(0.86-1.33)$ & $3.90(3.18-4.79)$ \\
\hline$\geqslant 65$ & $1.58(1.18-2.12)$ & $1.56(0.99-2.45)$ & $3.12(2.31-4.22)$ \\
\hline \multicolumn{4}{|l|}{ Gender } \\
\hline Female & $1.61(1.34-1.93)$ & $1.05(0.85-1.31)$ & $4.79(3.84-5.97)$ \\
\hline Male & $1.40(1.19-1.65)$ & $1.02(0.84-1.24)$ & $3.09(2.59-3.68)$ \\
\hline \multicolumn{4}{|l|}{ Presence of cardiometabolic risk factors ${ }^{a}$} \\
\hline Yes & $1.41(1.18-1.67)$ & $1.11(0.87-1.40)$ & $3.16(2.55-3.93)$ \\
\hline No & $1.58(1.34-1.87)$ & $1.05(0.88-1.26)$ & $4.14(3.47-4.94)$ \\
\hline
\end{tabular}



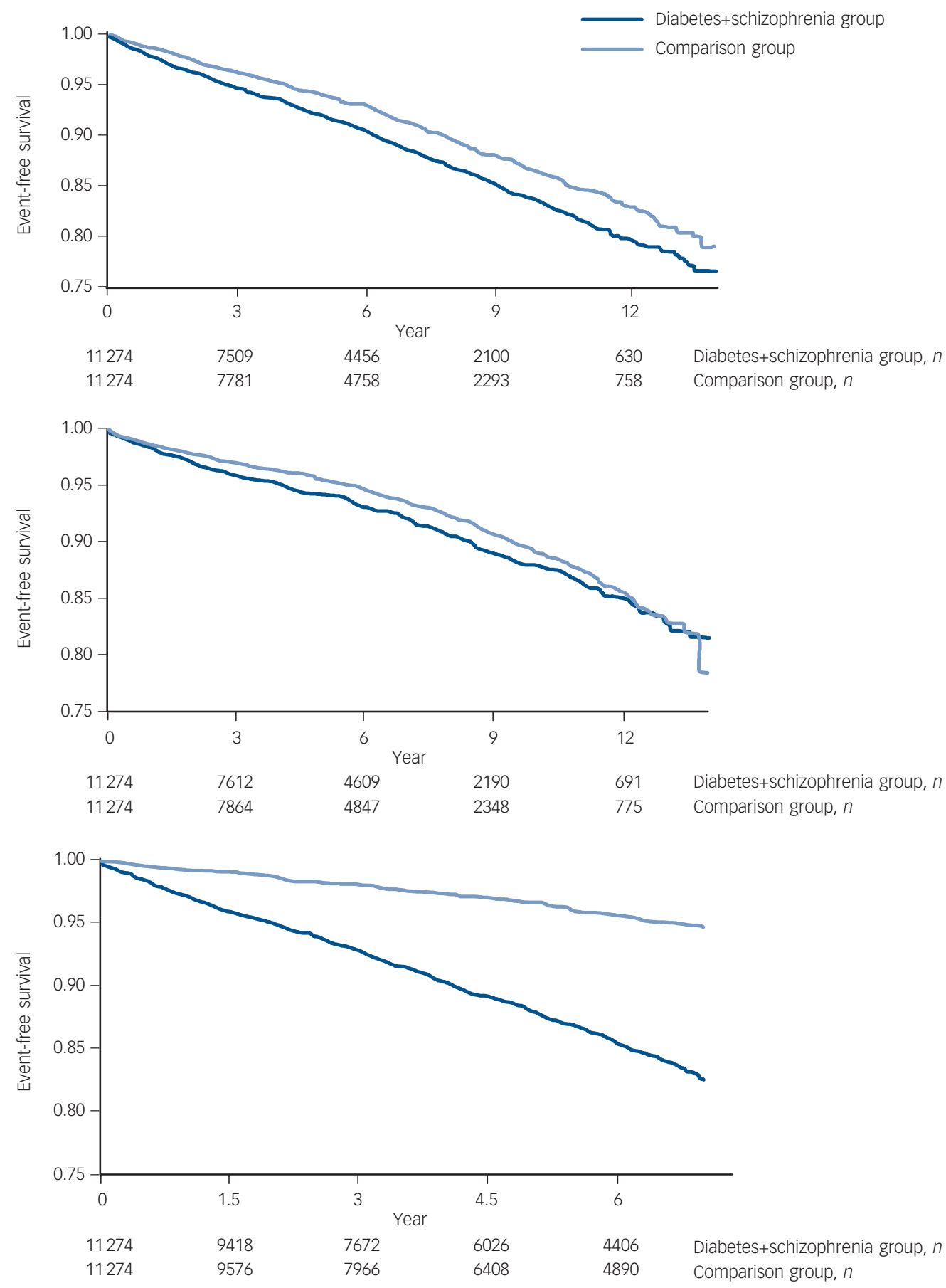

Fig. 2 Kaplan-Meier analysis of risk for (a) macrovascular complications, (b) microvascular complications and (c) all-cause mortality

different levels of adjustment are showed in online Table DS1 and the hazard ratios of covariates in online Table DS2.

Subgroup analyses revealed that the risk of mortality associated with schizophrenia was greater in women than in men $(P<0.001)$ but the risk of macrovascular or microvascular complications did not differ by gender. Age group or the presence of cardiometabolic risk factors did not modify the effect of schizophrenia on diabetic complications and mortality (all $P>0.5$ ). We further examined the quality of diabetes care during the follow-up periods and found that the diabetes+schizophrenia group had higher rates of good-quality indicators for receiving fasting blood glucose, lipid profile and serum creatinine testing, and better anti-diabetic medication adherence than those without schizophrenia. However, the diabetes+schizophrenia group were less likely to have had a retina examination (Table 3 ).

\section{Discussion}

This is one of the first studies using a cohort of patients with newly diagnosed diabetes to investigate the association between schizophrenia and advanced diabetes complications. The results showed that patients with diabetes and schizophrenia had a 1.49 -fold and a 3.68 -fold increased risk of macrovascular complications and all-cause mortality respectively. However, the risk of microvascular complications among the diabetes+schizophrenia 


\begin{tabular}{|c|c|c|c|}
\hline & \multicolumn{2}{|c|}{$n(\%)$} & \multirow[b]{2}{*}{$\begin{array}{l}\text { Adjusted OR } \\
(95 \% \mathrm{Cl})\end{array}$} \\
\hline & $\begin{array}{l}\text { Diabetes+schizophrenia group } \\
\qquad(n=11247)\end{array}$ & $\begin{array}{l}\text { Comparison group } \\
\qquad(n=11247)\end{array}$ & \\
\hline \multicolumn{4}{|l|}{ Examination } \\
\hline Fasting blood glucose & $7952(70.7)$ & $6814(60.6)$ & $1.22^{\mathrm{a}}(1.13-1.33)$ \\
\hline Haemoglobin $A_{1 c}$ & $3673(32.7)$ & $3239(28.8)$ & $1.08^{\mathrm{a}}(0.99-1.17)$ \\
\hline Lipid profiles & $5452(48.5)$ & $3911(34.8)$ & $1.37^{\mathrm{a}}(1.26-1.48)$ \\
\hline Urine protein profiles & $2116(18.8)$ & 1909 (17.0) & $1.00^{\mathrm{a}}(0.91-1.10)$ \\
\hline Serum creatinine & $5565(49.5)$ & 3508 (31.2) & $1.64^{\mathrm{a}}(1.52-1.78)$ \\
\hline Retina examination & $324(2.9)$ & $425(3.8)$ & $0.72^{\mathrm{a}}(0.58-0.89)$ \\
\hline Anti-diabetic medication adherence & & & $1.35^{\mathrm{b}}(1.23-1.47)$ \\
\hline None or diet & $2551(22.7)$ & $2571(22.9)$ & \\
\hline Poor adherence & $3221(28.6)$ & $3917(34.8)$ & \\
\hline Good adherence & $5475(48.6)$ & $4759(42.3)$ & \\
\hline
\end{tabular}

group was not significantly different from the patients with diabetes but not schizophrenia.

Our findings were generally in line with a cohort study using linked data-sets in Western Australia, which showed patients with schizophrenia had a significantly increased risk of mortality and an increased trend for diabetes complications. ${ }^{13}$ The marked increased mortality rate of patients with diabetes and schizophrenia was also compatible with previous studies that showed that patients with schizophrenia have a two- to fourfold increased mortality rate because of endocrine, cardiovascular and genitourinary diseases, compared with the general population. ${ }^{23}$ However, our results were contradictory to those found by a cohort study using Medicaid and Medicare claims data in Massachusetts, USA. ${ }^{14}$ Leung and colleagues found that patients with schizophrenia or paranoid state had lower rates of adverse diabetes outcomes than patients without mental disorders. ${ }^{14}$ Their findings might be markedly biased by a lack of control for disease duration of diabetes because the mean age of the patients with schizophrenia (52 years old, s.d. $=13$ ) was much younger than that of patients without mental illnesses (70 years old, s.d. $=13)$, who would have a longer disease duration and a higher risk for diabetes complications.

Although the findings of significantly increased risks for all-cause mortality and macrovascular complications among patients with schizophrenia lent evidence to support our hypothesis, the lack of increased risk for microvascular complications was unexpected. One possible explanation is that patients with schizophrenia might die as a result of competing causes, such as unnatural death and infectious diseases, ${ }^{23}$ before the development of diabetes complications. Another possible explanation is that patients with diabetes and schizophrenia may have similar levels of glycaemic control to the general diabetes population. Intensive glycaemic control is the key factor to reduce the development or microvascular complications. ${ }^{2}$ Three cross-sectional studies showed that the $\mathrm{HbA}_{1 \mathrm{c}}$ level in patients with schizophrenia was similar to or lower than that in those without severe mental illnesses. ${ }^{9-11}$ Although one retrospective cohort study showed that patients with schizophrenia were more likely to be admitted to hospital or visit emergency department for hyperglycaemia or hypoglycaemia, ${ }^{12}$ this study did not distinguish hyperglycaemia from hypoglycaemia. Patients with diabetes and schizophrenia might have lower average blood glucose, thereby being associated with higher risk for hypoglycaemia rather than hyperglyacemia. There is little evidence to suggest that patients with diabetes and schizophrenia have poorer glycaemic control than those without schizophrenia.

The risk of macrovascular complications might not be reduced by intensive glycaemic control. ${ }^{24}$ Risk factors, such as smoking, hypertension or dyslipidaemia might be more influential. ${ }^{24,25}$ Individuals with schizophrenia have a higher risk of unhealthy lifestyle behaviours, such as tobacco smoking or physical inactivity. ${ }^{26}$ Although we could not directly measure lifestyle behaviours in this study, we found that patients with schizophrenia had a higher prevalence of dyslipidaemia, alcoholor substance-related disorders, as well as chronic pulmonary diseases, which are related to tobacco smoking. These unhealthy lifestyle behaviours might be important contributors to the macrovascular complications and mortality in patients with both diabetes and schizophrenia.

In the subgroup analysis, we found that women had a greater risk for mortality associated with schizophrenia than did men. Previous studies have shown that the effect of diabetes on the risk of coronary disease mortality is significantly greater for women than men. ${ }^{27}$ Furthermore, the adverse changes in lipid profile and blood pressure were more obvious in women with diabetes than those in men with diabetes. ${ }^{28}$ Therefore, there might be a synergistic effect of schizophrenia and female gender on the risk of mortality. Schizophrenia per se might directly increase the risk of diabetes complications. Schizophrenia is a link to dysregulation of the hypothalamic-pituitary-adrenal axis. ${ }^{29}$ Increased cortisol secretion has also been reported to be related to diabetes complications. ${ }^{30}$ Thus, the abnormality of the hypothalamicpituitary-adrenal axis might be one of the possible mechanisms for the associations between schizophrenia and diabetes complications. In addition, inflammatory cytokines associated with the pathogenesis of schizophrenia ${ }^{31}$ might also further increase the risk of diabetes complications. ${ }^{32}$

Use of antipsychotics is associated with elevated blood glucose level and the development of diabetes among patients with schizophrenia. ${ }^{33}$ In addition, antipsychotic exposure might be associated with stroke and cardiovascular adverse effect. ${ }^{34,35}$ However, antipsychotic treatment can improve psychosocial functioning and self-care behaviours in patients with schizophrenia, thereby having beneficial effects on health outcomes. Evidence shows that long-term antipsychotic treatment is associated with lower mortality than that found in those not receiving this treatment. ${ }^{36}$ Thus, the role of antipsychotic drugs in the progression of diabetes complications remains unclear and warrants further investigation. 


\section{Quality of diabetes care}

Could patients with diabetes and schizophrenia have poorer quality of diabetes care, thereby having a higher risk of macrovascular complications and mortality? Several studies have shown that severe mental illnesses might have a negative impact on the quality of diabetes care. ${ }^{37,38}$ However, in this study, we found most of the quality indicators of diabetes care for patients with schizophrenia were better than or equal to that for patients without schizophrenia, except the rate of retina examination. These findings are in line with previous studies that showed that there was little difference between patients with diabetes with and without schizophrenia in terms of the rates of receipt for tests for detecting diabetes complications. ${ }^{10,39}$ Furthermore, we found that patients with diabetes and schizophrenia had better antidiabetic medication adherence than those without schizophrenia, which is compatible with one cross-sectional survey using Veterans Affairs health system administrative data. ${ }^{40}$ The quality of diabetes care in patients with schizophrenia varies across healthcare systems. ${ }^{10,11,38-40}$ Taiwan's universal NHI, with the characteristics of very low copayment and high utilisation rate, greatly reduces health disparities. ${ }^{41}$ Moreover, patients with schizophrenia can get anti-diabetic medications and screening tests in psychiatric settings in Taiwan. Therefore, the quality of diabetes care was not an important contributing factor for poor clinical outcome among patients with schizophrenia.

\section{Strengths and limitations}

The strengths of this study are the novelty of analysing the associations between schizophrenia and diabetes complications, the use of a nationwide representative cohort with patients newly diagnosed with diabetes, clear temporal relationships, a very large sample and a well-defined method for identifying complications of diabetes. However, several limitations should be considered. First, patients might have untreated hyperglycaemia for a long period before the diagnosis of diabetes. Given that diabetes mellitus has an insidious onset and may not be diagnosed immediately, the disease duration of diabetes mellitus is likely to be underestimated. In this study, we found that patients with schizophrenia utilised healthcare systems more frequently than those without schizophrenia; therefore, patients with schizophrenia have more opportunities for diabetes screening thereby being diagnosed at an earlier stage of diabetes mellitus. If this is the case, the risk of complications and mortality in patients with diabetes and schizophrenia might be underestimated. Second, we identified and confirmed patients with schizophrenia based on in-patient claims records. Patients with schizophrenia who were treated only in out-patient settings were not enrolled in the study; therefore, we could not generalise our findings to patients with schizophrenia of mild severity. Third, the accuracy of diagnosis of microvascular complications and peripheral vascular diseases was not validated in the NHIRD; however, we used procedure claims to confirm the ambulatory diagnostic code or used only in-patient claims to minimise the possibility of misclassifications. Fourth, information for the causes of mortality in the NHIRD were not available. The role of diabetes in the high mortality rate in schizophrenia remains unclear. Further research should explore the relationship between diabetes and cause-specific mortality among patients with schizophrenia. Finally, several important clinical factors, such as age at schizophrenia onset, were not measured in this study because of the short observational period prior to the diabetes diagnosis. In addition, other factors, such as education, smoking, body weight or exercise, were also not available from the NHIRD. The roles of these factors on the associations between schizophrenia and diabetes complications warrant further investigation.

\section{Clinical implications and further research}

Patients with diabetes have a higher risk of macrovascular complications and mortality if they are also have schizophrenia. However, the quality of diabetes care among patients with schizophrenia was better than or equal to that of those without schizophrenia. Unhealthy lifestyle behaviours might be important contributors to poor diabetes outcomes among people with schizophrenia. Of note, patients with schizophrenia might be less likely to receive standard diabetes education and cues from health providers about glucose monitors. ${ }^{38}$ Therefore, lifestyle interventions for exercise, diet control and tobacco cessation should be emphasised. Further research should also focus on effective interventions to reduce diabetes complications in patients with schizophrenia.

Chi-Shin Wu, MD, MSc, Department of Psychiatry, Far Eastern Memorial Hospital, Taipei, Graduate Institute of Epidemiology and Preventive Medicine, National Taiwan University College of Public Health, Taipei and Department of Psychiatry, National Taiwan University Hospital and College of Medicine, Taipei, Taiwan; Mei-Shu Lai, MD, $\mathrm{PhD}$, Graduate Institute of Epidemiology and Preventive Medicine, National Taiwan University College of Public Health, Taipei, Taiwan; Susan Shur-Fen Gau, MD, PhD, Graduate Institute of Epidemiology and Preventive Medicine, National Taiwan University College of Public Health, Taipei and Department of Psychiatry, National Taiwan University Hospital and College of Medicine, Taipei, Taiwan

Correspondence: Susan Shur-Fen Gau, Department of Psychiatry, National Taiwan University Hospital \& College of Medicine, No. 7, Chung-Shan South Road, Taipei 10002, Taiwan. Email: gaushufe@ntu.edu.tw

First received 22 Dec 2013, final revision 30 oct 2014, accepted 9 Dec 2014

\section{Funding}

This work was supported by grants from Far Eastern Memorial Hospital (FEMH-2013-C-005), and from National Bureau of Controlled Drugs, Department of Health (NBCD-97014), Taiwan. The Far Eastern Memorial Hospital and Taiwan's National Bureau of Controlled Drugs had no role in the design and conduct of the study; in the collection, analysis, and interpretation of the data; or in the preparation, review or approval of the manuscript.

\section{Acknowledgements}

This study is based in part on data from the National Health Insurance Research Database provided by the Bureau of National Health Insurance of the Department of Health, Taiwan, and managed by National Health Research Institutes, Taiwan. The interpretation and conclusions contained in this article do not represent those of the Bureau of National Health Insurance, the Department of Health, or the National Health Research Institutes.

\section{References}

1 Stratton IM, Adler Al, Neil HA, Matthews DR, Manley SE, Cull CA, et al. Association of glycaemia with macrovascular and microvascular complications of type 2 diabetes (UKPDS 35): prospective observational study. BMJ 2000; 321: 405-12.

2 UK Prospective Diabetes Study (UKPDS) Group. Intensive blood-glucose control with sulphonylureas or insulin compared with conventional treatment and risk of complications in patients with type 2 diabetes (UKPDS 33). UK Prospective Diabetes Study (UKPDS) Group. Lancet 1998; 352: 837-53.

3 Dixon L, Weiden P, Delahanty J, Goldberg R, Postrado L, Lucksted A, et al. Prevalence and correlates of diabetes in national schizophrenia samples. Schizophr Bull 2000; 26: 903-12.

4 Holt Rl, Bushe C, Citrome L. Diabetes and schizophrenia 2005: are we any closer to understanding the link? J Psychopharmacol 2005; 19: 56-65.

5 Bai YM, Su TP, Chen MH, Chen TJ, Chang WH. Risk of developing diabetes mellitus and hyperlipidemia among patients with bipolar disorder, major depressive disorder, and schizophrenia: a 10-year nationwide populationbased prospective cohort study. J Affect Disord 2013; 150: 57-62.

6 Holt Rl, Peveler RC, Byrne CD. Schizophrenia, the metabolic syndrome and diabetes. Diabet Med 2004; 21: 515-23. 
7 Newcomer JW, Haupt DW, Fucetola R, Melson AK, Schweiger JA, Cooper BP, et al. Abnormalities in glucose regulation during antipsychotic treatment of schizophrenia. Arch Gen Psychiatry 2002; 59: 337-45.

8 Sernyak MJ, Leslie DL, Alarcon RD, Losonczy MF, Rosenheck R. Association of diabetes mellitus with use of atypical neuroleptics in the treatment of schizophrenia. Am J Psychiatry 2002; 159: 561-6.

9 Dixon LB, Kreyenbuhl JA, Dickerson FB, Donner TW, Brown CH, Wohlheiter K, et al. A comparison of type 2 diabetes outcomes among persons with and without severe mental illnesses. Psychiatr Serv 2004; 55: 892-900.

10 Krein SL, Bingham CR, McCarthy JF, Mitchinson A, Payes J, Valenstein M. Diabetes treatment among VA patients with comorbid serious mental illness. Psychiatr Serv 2006; 57: 1016-21.

11 Weiss AP, Henderson DC, Weilburg JB, Goff DC, Meigs JB, Cagliero E, et al. Treatment of cardiac risk factors among patients with schizophrenia and diabetes. Psychiatr Serv 2006; 57: 1145-52.

12 Becker T, Hux J. Risk of acute complications of diabetes among people with schizophrenia in Ontario, Canada. Diabetes Care 2011; 34: 398-402.

13 Mai Q, Holman CD, Sanfilippo FM, Emery JD, Preen DB. Mental illness related disparities in diabetes prevalence, quality of care and outcomes: a population-based longitudinal study. BMC Med 2011; 9: 118.

14 Leung G, Zhang J, Lin WC, Clark RE. Behavioral disorders and diabetes-related outcomes among Massachusetts Medicare and Medicaid beneficiaries. Psychiatr Serv 2011; 62: 659-65.

15 WHO Collaborating Centre for Drug Statistic Methodology. Guidelines for ATC Classification and DDD Assignment. WHO, 2009.

16 Cheng $\mathrm{CL}$, Kao YH, Lin SJ, Lee CH, Lai ML. Validation of the National Health Insurance Research Database with ischemic stroke cases in Taiwan Pharmacoepidemiol Drug Saf 2011; 20: 236-42.

17 Lin CC, Lai MS, Syu CY, Chang SC, Tseng FY. Accuracy of diabetes diagnosis in health insurance claims data in Taiwan. J Formos Med Assoc 2005; 104 157-63.

18 Wu CY, Chan FK, Wu MS, Kuo KN, Wang CB, Tsao CR, et al. Histamine2receptor antagonists are an alternative to proton pump inhibitor in patients receiving clopidogrel. Gastroenterology 2010; 139: 1165-71.

19 World Health Organization. International Classification of Diseases, Ninth Revision, Clinical Modification (ICD-9-CM). WHO, 2009.

20 Chang $\mathrm{CH}$, Shau WY, Jiang YD, Li HY, Chang TJ, Sheu WH, et al. Type 2 diabetes prevalence and incidence among adults in Taiwan during 19992004: a national health insurance data set study. Diabet Med 2010; 27 : 636-43.

21 Lin DY, Wei L, Ying Z. Checking the Cox model with cumulative sums of Martingale-based residuals. Biometrika 1993; 80: 557-72.

22 Cramer JA, Roy A, Burrell A, Fairchild CJ, Fuldeore MJ, Ollendorf DA, et al. Medication compliance and persistence: terminology and definitions. Value Health 2008; 11: 44-7.

23 Saha S, Chant D, McGrath J. A systematic review of mortality in schizophrenia: is the differential mortality gap worsening over time? Arch Gen Psychiatry 2007; 64: 1123-31.

24 Skyler JS, Bergenstal R, Bonow RO, Buse J, Deedwania P, Gale EA, et al. Intensive glycemic control and the prevention of cardiovascular events: implications of the ACCORD, ADVANCE, and VA diabetes trials: a position statement of the American Diabetes Association and a scientific statement of the American College of Cardiology Foundation and the American Heart Association. Diabetes Care 2009; 32: 187-92.

25 Eliasson B. Cigarette smoking and diabetes. Prog Cardiovasc Dis 2003; 45 405-13.

26 Brown S, Birtwistle J, Roe L, Thompson C. The unhealthy lifestyle of people with schizophrenia. Psychol Med 1999; 29: 697-701.

27 Lee WL, Cheung AM, Cape D, Zinman B. Impact of diabetes on coronary artery disease in women and men: a meta-analysis of prospective studies. Diabetes Care 2000; 23: 962-8.

28 Howard BV, Cowan LD, Go O, Welty TK, Robbins DC, Lee ET. Adverse effects of diabetes on multiple cardiovascular disease risk factors in women. The Strong Heart Study. Diabetes Care 1998; 21: 1258-65.

29 Walker E, Mittal V, Tessner K. Stress and the hypothalamic pituitary adrenal axis in the developmental course of schizophrenia. Annu Rev Clin Psychol 2008; 4: 189-216.

30 Chiodini I, Adda G, Scillitani A, Coletti F, Morelli V, Di Lembo S, et al. Cortisol secretion in patients with type 2 diabetes: relationship with chronic complications. Diabetes Care 2007; 30: 83-8.

31 Leonard BE, Schwarz M, Myint AM. The metabolic syndrome in schizophrenia: is inflammation a contributing cause? J Psychopharmacol 2012; 26: 33-41.

32 King GL. The role of inflammatory cytokines in diabetes and its complications. J Periodontol 2008; 79: 1527-34.

33 Haupt DW, Newcomer JW. Hyperglycemia and antipsychotic medications. I Clin Psychiatry 2001; 62 (suppl 27): 15-26, discussion 40-1.

34 Wu CS, Wang SC, Gau SS, Tsai HJ, Cheng YC. Association of stroke with the receptor-binding profiles of antipsychotics-a case-crossover study. Biol Psychiatry 2013; 73: 414-21.

35 Buckley NA, Sanders P. Cardiovascular adverse effects of antipsychotic drugs. Drug Saf 2000; 23: 215-28.

36 Tiihonen J, Lonnqvist J, Wahlbeck K, Klaukka T, Niskanen L, Tanskanen A et al. 11-year follow-up of mortality in patients with schizophrenia: a population-based cohort study (FIN11 study). Lancet 2009; 374: 620-7.

37 Mitchell AJ, Malone D, Doebbeling CC. Quality of medical care for people with and without comorbid mental illness and substance misuse: systematic review of comparative studies. Br J Psychiatry 2009; 194: 491-9.

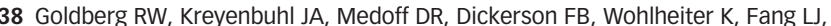
et al. Quality of diabetes care among adults with serious mental illness. Psychiatr Serv 2007; 58: 536-43.

39 Desai MM, Rosenheck RA, Druss BG, Perlin JB. Mental disorders and quality of diabetes care in the veterans health administration. Am J Psychiatry 2002; 159: $1584-90$.

40 Kreyenbuhl J, Dixon LB, McCarthy JF, Soliman S, Ignacio RV, Valenstein M. Does adherence to medications for type 2 diabetes differ between individuals with vs without schizophrenia? Schizophr Bull 2010; 36: 428-35.

41 Wen CP, Tsai SP, Chung WS. A 10-year experience with universal health insurance in Taiwan: measuring changes in health and health disparity. Ann Intern Med 2008; 148: 258-67. 